\title{
Retinoblastoma en pediatría, experiencia en un hospital pediátrico
}

\author{
ARTURO TRINCADO M. ${ }^{1}$, JUAN PABLO LÓPEZ G. ${ }^{1}$, MILITZA GONZÁLEZ N. ${ }^{1}$, \\ EDUARDO VILLASECA D. ${ }^{1}$, ALEJANDRA ROIZEN B. ${ }^{1}$, DENISE MANIEU M. ${ }^{1}$, \\ JOSÉ DÍAZ C. ${ }^{2}$, MILENA VILLARROEL C. ${ }^{2}$, ALEJANDRA HENRÍQUEZ V. ${ }^{3}$, \\ JUAN JOSÉ LATORRE L. ${ }^{3}$, SERGIO GALANO TRIVIÑO ${ }^{4}$ \\ 1. Servicio de Oftalmología Hospital Luis Calvo Mackenna. \\ 2. Servicio de Oncología Hospital Luis Calvo Mackenna. \\ 3. Servicio de Anatomía Patológica Hospital Luis Calvo Mackenna. \\ 4. Servicio de Oftalmología Hospital Del Salvador.
}

\begin{abstract}
Retinoblastoma in children, experience at a pediatric hospital

Methods: Retrospective analysis of clinical charts of 41 children (59 eyes) diagnosed with retinoblastoma and treated by a multidisciplinary team at Hospital Luis Calvo Mackenna in Santiago-Chile, between 1999 and 2007. The information included gender, laterality, diagnosis age, presenting signs, tumor spread, treatment modality and survival rate. Results: A total of 23 cases $(56 \%)$ were unilateral and 18 cases (44\%) were bilateral. The mean age at diagnosis was 21.6 months (range $2-84$ ) and 27 children $(65.9 \%)$ were male. The most common presenting signs were leucokoria $(51.2 \%)$, strabismus $(24.4 \%)$ and proptosis $(4.9 \%)$. Enucleation was performed in 48 eyes $(81.3 \%)$, being the only required treatment in 17 children (41.5\%). The remaining 24 patients received systemic and/or local therapy with chemotherapy, focal therapy and external beam radiation. 5 children died during the follow - up study period, due to extraocular extension to the orbit, central nervous system and bone marrow. Conclusion: In spite of high enucleation rate as initial therapy for retinoblastoma, the survival rate with this current treatment protocol is similar to those from developed countries.

(Key words: Retinoblastoma, treatment, chemotherapy).

Rev Chil Pediatr 2008; 79 (6): 614-622
\end{abstract}

\section{RESUMEN}

Se presenta un estudio retrospectivo de las fichas clínicas de 41 niños (59 ojos) con diagnóstico de retinoblastoma tratados por un equipo multidisciplinario en el Hospital Luis Calvo Mackenna, Santiago, Chile, entre los años 1999-2007. Se recolectó información respecto al género, edad al diagnóstico, signos de presentación, lateralidad, diseminación del tumor, tipos de tratamiento y sobrevida. Veintitrés casos (56\%)

Trabajo recibido el 09 de octubre de 2008, devuelto para corregir el 01 de noviembre de 2008, segunda versión el 04 de diciembre de 2008, aceptado para publicación el 10 de diciembre de 2008. 
fueron unilaterales y $18(44 \%)$ bilaterales. La edad promedio al momento del diagnóstico fue de 21,6 meses (rango 2-84) y 27 niños $(65,9 \%)$ fueron hombres. Los signos de presentación más frecuentes fueron leucocoria $(51,2 \%)$, estrabismo $(24,4 \%)$ y proptosis $(4,9 \%)$. Se realizó enucleación en 48 ojos afectados $(81,3 \%)$, siendo el único tratamiento necesario en 17 niños $(41,5 \%)$. Los 24 pacientes restantes recibieron tratamientos complementarios locales y/o sistémicos en la modalidad de quimioterapia, terapia focal y radioterapia externa. Durante el período de seguimiento del estudio fallecieron 5 niños, todos ellos con extensión extraocular de la enfermedad hacia la órbita, sistema nervioso central o médula ósea. Conclusiones: No obstante el alto porcentaje de pacientes con retinoblastoma que requieren enucleación como terapia inicial, la tasa de sobrevida con el protocolo actual de tratamiento es comparable a la de paises desarrollados.

(Palabras clave: Retinoblastoma, tratamiento, quimioterapia).

Rev Chil Pediatr 2008; 79 (6): 614-622

\section{Introducción}

El retinoblastoma $(\mathrm{RB})$ es el cáncer ocular más frecuente en los niños, presentándose con una incidencia de 1 en 15.000 a 1 en 20.000 nacidos vivos ${ }^{1}$. La enfermedad es invariablemente mortal sin tratamiento y sus consecuencias visuales y psicosociales pueden ser desvastadoras. En un país como el nuestro donde nacen unos 230.831 recién nacidos vivos por año (MINSAL 2005, http://163.247.51.54/ rem2007/natalidad/region_gen_2005nacim.php) se presentan, según los últimos datos publicados del Programa Infantil de Drogas Antineoplásicas PINDA, aproximadamente $13 \mathrm{ca}-$ sos nuevos de RB por año (http://www.minsal.cl/ ici/guiasclinicas/Linfomani\%F1osR Mayo10. pdf), siendo la mayoría de ellos atendidos en el sistema público de salud. El Hospital Luis Calvo Mackenna (HLCM) es un centro de referencia oncológico pediátrico del sistema público de nuestro país que brinda atención oncológica y oftalmológica a gran parte de los niños con RB. Por tratarse de una enfermedad de baja ocurrencia resulta muy difícil comparar la eficacia de los diferentes protocolos de tratamiento de RB que se aplican en los diversos centros del mundo ${ }^{2}$ y en especial en un país como Chile en donde la enfermedad se concentra en unos pocos centros de referencia. Existe además escasa información nacional publicada y actualizada sobre la experiencia oftalmológica en el manejo del RB, siendo la mayoría revisiones de casos en diferentes momentos históricos y basados, por lo tanto, en diferentes protocolos históricos de tratamiento ${ }^{3,4}$, por lo que se hace muy necesario contar con resultados nacionales actualizados sobre el manejo del RB.

Con el objeto analizar la situación epidemiológica actual del RB en nuestro medio y evaluar los resultados oftalmológicos y oncológicos en esta patología, se realiza una revisión de nuestra experiencia en una serie de pacientes pediátricos portadores de Retinoblastoma.

\section{Pacientes y Métodos}

Se presenta una serie retrospectiva de los niños con diagnóstico de RB ingresados al Servicio de Oncología y/u Oftalmología del Hospital Luis Calvo Mackenna entre el 01 de Enero de 1999 y el 31 de Diciembre de 2007. Para cada paciente se consignó su fecha de nacimiento, género, procedencia geográfica, edad al inicio de los signos y síntomas, motivo de consulta, fechas de primera consulta, fecha de primera atención en el hospital Luis Calvo Mackenna y edad al momento del diagnóstico definitivo. Respecto a la enfermedad se registraron los antecedentes familiares, lateralidad y número de tumores, etapificación según clasificación de Reese-Ellsworth, anatomía patológica de los ojos enucleados, tipo de quimioterapia recibida y los procedimientos terapéuticos oftalmológicos efectuados. Finalmente, se investigó el número de reactivaciones tumorales, aparición de nuevos tumores y diseminación a distancia. La información de las fichas clínicas se complementó con datos adicionales obtenidos de las interconsultas de derivación de los pacientes y entrevistas con sus padres y/o tuto- 
res. Los datos se tabularon y analizaron utilizando el software Excel de Microsoft. Se utilizó medidas de tendencia central y dispersión según correspondiese. Esta revisión contó con la aprobación del comité de ética del HLCM para la revisión de las fichas clínicas y difusión de los resultados.

\section{Resultados}

Entre los años 1999 y 2007 ingresaron al hospital Luis Calvo Mackenna 46 pacientes con diagnóstico de Retinoblastoma, cinco de los cuales presentaban fichas incompletas por lo que fueron excluidos del estudio, quedando la muestra constituida por un total de 41 niños y 59 ojos afectados, correspondiendo a 23 niños $(56 \%)$ con tumores unilaterales y 18 niños (44\%) con tumores bilaterales (36 ojos). El número de ingresos por año fue variable con un promedio de 4,56 casos nuevos por año en promedio y con un rango de casos entre 2 (años 2000 y 2007) y 8 (años 2002 y 2003).

El tiempo de seguimiento promedio fue de 55,7 meses (rango 5-105).

\section{Características demográficas}

La mayoría de los pacientes fueron hombres $(27,65,9 \%)$ y el promedio de edad al ingreso fue de 21,6 meses (rango 2-84). Treinta y ocho casos $(92,68 \%)$ se diagnosticaron antes

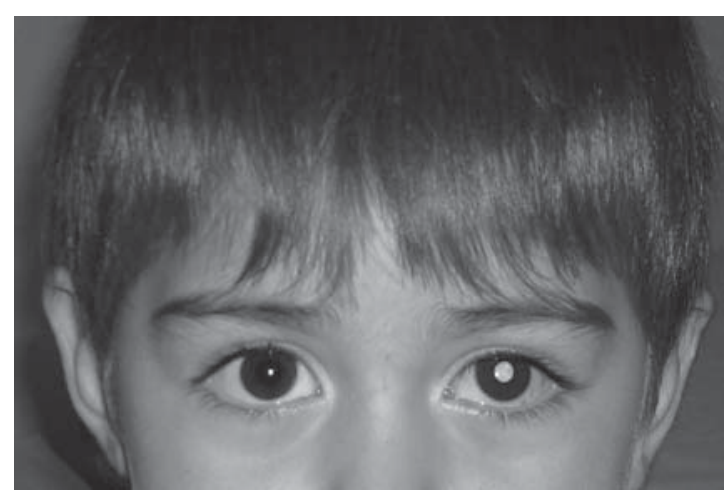

Figura 1. Leucocoria (pupila blanca) izquierda en uno de los pacientes del estudio, más evidente en una fotografía con flash. de los 4 años. Sólo 2 casos (4,9\%) se diagnosticaron antes de los 2 meses de edad y un caso a $\operatorname{los} 7$ años. Los casos bilaterales se diagnosticaron más precozmente que los unilaterales con un promedio de edad de 15,7 meses (rango 279) comparado con 28 meses (rango 8-84) respectivamente. Trece pacientes $(31,7 \%)$ provenían de la región Metropolitana, seguidas de las regiones II, VII y VIII (cada una con 4 pacientes, 9,8\%), en tanto que las regiones III, XI y XII no registraron derivaciones.

\section{Características Clínicas}

\section{Historia Familiar}

En sólo 1 de los 41 pacientes se encontró consignado en su ficha la presencia de otros familiares afectados con RB, correspondiendo a un caso de un niño con RB unilateral. Por el contrario, los 18 casos bilaterales de la presente serie, considerados en la práctica como portadores de la mutación germinal y por consiguiente con alta probabilidad de historia familiar de $\mathrm{RB}$, no tenían historia de familiares afectados por la enfermedad, contrastando este hecho con la literatura ${ }^{5}$.

\section{Motivo de Consulta}

El motivo de consulta más frecuente fue la leucocoria (brillo blanco en la pupila) y el estrabismo. Figura 1 y tabla 1.

El intervalo de tiempo promedio transcurrido entre la observación de los primeros signos

Tabla 1. Motivo de Consulta en 41 niños con retinoblastoma

\begin{tabular}{lrc}
\hline Signo & n niños & (\%) \\
\hline Leucocoria & 21 & $(51,2)$ \\
Estrabismo & 10 & $(24,4)$ \\
Leucocoria + estrabismo & 2 & $(4,9)$ \\
Proptosis & 2 & $(4,9)$ \\
No consignado & 2 & $(4,9)$ \\
Disminución de visión & 1 & $(2,4)$ \\
Ojo rojo & 1 & $(2,4)$ \\
Exoftalmos inflamatorio & 1 & $(2,4)$ \\
Hallazgo de examen (TAC) & 1 & $(2,4)$ \\
Total & 41 & $(100)$ \\
\hline
\end{tabular}


por parte de los padres y la primera consulta con cualquier profesional de la salud fue de 128,7 días (rango 1-545) en tanto que entre la primera consulta y la primera evaluación oftalmológica fue de 40,4 días (1-250) en promedio. El tiempo entre la primera evaluación oftalmológica y la evaluación en el Hospital Luis Calvo Mackenna fue en promedio 6,7 días (1-40). La mayoría de las derivaciones al HLCM fue realizada por oftalmólogos (37 casos, 90,3\%), y pediatras (3 casos, $7,3 \%)$. Un caso $(2,4 \%)$ fue derivado por un médico general. De gran importancia resulta el hecho que de los 37 pacientes derivados por oftalmólogo, 9 casos (22\%) resultaron ser sindromes enmascarados lo que significa que fueron derivados con otro diagnóstico presuntivo, comprobándose finalmente la presencia de RB en nuestro hospital. Los diagnósticos de derivación de estos niños fueron enfermedad de Coats (2 casos), hifema (1), desprendimiento de retina (1), celulitis orbitaria (1), proptosis inespecífica (1), estrabismo (1), uveitis (1) y catarata con glaucoma (1).

\section{Lateralidad y Focalidad}

Encontramos una proporción similar entre casos uni $(56 \%)$ y bilaterales (44\%). Dos casos unilaterales se presentaron como multifocales (más de un tumor identificable en la retina), representando probablemente niños portadores de mutación germinal para retinoblastoma ${ }^{6}$. Tabla 2.

\section{Número y Localización de los tumores}

Durante el período del estudio 48 de 59 ojos fueron enucleados por presentar enfermedad muy avanzada, correspondiendo a 23 ojos de niños con tumores unilaterales, 18 ojos de niños con tumores bilaterales a quienes se les enucleó inicialmente un ojo y 7 ojos adicionales de este mismo grupo de niños que finalmente requirieron enucleación bilateral. En los ojos que fueron enucleados resultó muy difícil identificar oftalmoscópicamente la focalidad (número) y localización de los tumores previo a la enucleación ya que la mayoría presentaba una gran masa tumoral que comprometía la casi totalidad de la cámara vítrea y/o tenía un desprendimiento de retina total. La histopatología presentó las mismas dificultades en cuanto a la identificación de focalidad y número de tumores dada la extensión masiva de la enfermedad que imposibilita precisar el número de tumores.

En contraste, fue posible determinar con precisión la localización anatómica de los tumores presentes en los 18 ojos inicialmente conservados de los niños con RB bilateral a quienes se les enucleó uno de sus ojos. La mayoría de los tumores se presentó en el polo posterior del ojo ( 6 ojos; $33,3 \%$ ), en el polo posterior y la periferia media retinal $(5 ; 27,8 \%)$, en la periferia media retinal $(5 ; 27,8 \%)$, en la periferia media y periferia extrema $(2 ; 11 \%)$ y en ningún caso los tumores se presentaron en la periferia extrema exclusiva. Esta información tiene relevancia clínica, pues resulta mucho más probable detectar los tumores retinales que se ubican en el polo posterior ya que pueden sospecharse más fácilmente mediante el examen del rojo pupilar, aumentando el nivel de dificultad en el diagnóstico con este examen a medida que los tumores se alejan del nervio óptico hacia la periferia retinal donde sólo es posible detectarlos mediante el examen de fondo de ojos con dilatación pupilar y depresión escleral. En 5 de los 11 ojos con tumores ubicados en el polo posterior existía compromiso macular, en 1 del nervio óptico, en 3 ojos tanto de la mácula como del nervio óptico y en sólo 2 casos no existía

Tabla 2. Lateralidad y focalidad de tumores en 41 niños con retinoblastoma

\begin{tabular}{|c|c|c|c|}
\hline Lateralidad & $\begin{array}{c}\text { Unifocal } \\
\text { n niños (\%) }\end{array}$ & $\begin{array}{l}\text { Multifocal } \\
\text { n niños (\%) }\end{array}$ & $\begin{array}{c}\text { Total } \\
\text { n niños }(\%)\end{array}$ \\
\hline Unilateral & $21 \quad(77,8)$ & $(14,3)$ & $(56,1)$ \\
\hline Bilateral $(*)$ & $6 \quad(22,2)$ & $12 \quad(85,7)$ & $(43,9)$ \\
\hline Total & $27 \quad(100)$ & $14(100)$ & $41 \quad(100)$ \\
\hline
\end{tabular}

(*) La focalidad (número de tumores) en RB bilateral se consignó en el ojo conservado (ver texto). 
compromiso macular ni de nervio óptico. El compromiso de la mácula significa invariablemente daño a la visión central del paciente, en tanto que el compromiso del nervio óptico puede implicar extensión tumoral al sistema nervioso central. Figura 2.

\section{Clasificación Clínica de los tumores}

Las recomendaciones actuales sugieren clasificar los RB utilizando la Clasificación Internacional para Retinoblastoma Intraocular

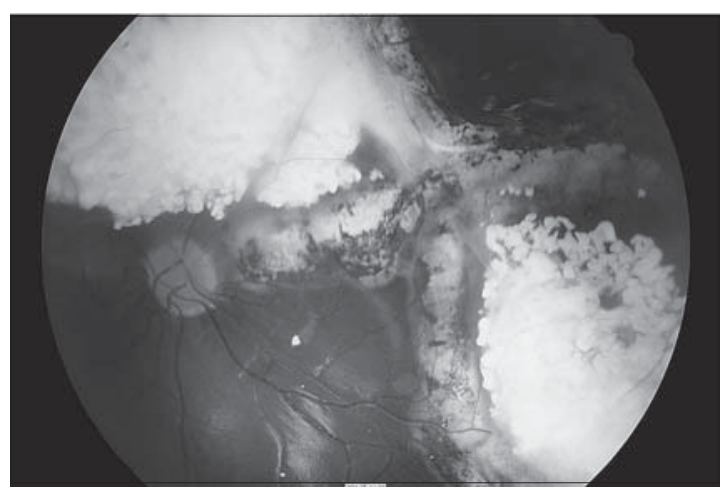

Figura 2. Retinoblastoma multifocal (2 tumores) con compromiso de la mácula y cercanía al nervio óptico en un paciente del estudio. Obsérvese la presencia de calcificaciones visibles como granulación blanquecina en los tumores y la pigmentación oscura cicatrizal post tratamiento con LASER. $(\text { IIRC })^{7}$ desarrollada para predecir resultados con las técnicas terapéuticas actuales (principalmente quimioterapia y tratamiento focal) no obstante, a fin de poder comparar nuestros resultados con reportes previos nacionales e internacionales, usamos para nuestros pacientes la clasificación de Reese-Ellsworth, ampliamente difundida en la literatura internacional y creada en la década de 1960 para predecir pronóstico cuando el RB era tratado con radioterapia externa. Tabla 3. En el 92,7\% de los pacientes el primer tratamiento efectuado fue la enucleación, presentando la mayoría de los ojos compromiso de casi la totalidad de la cámara vítrea (etapa V). Por el contrario, los ojos conservados correspondieron a etapas más precoces (I, II y III) con tumores más pequeños y de más fácil tratamiento.

\section{Modalidades de Tratamiento Utilizado}

\section{Enucleación y Terapia Focal}

En 38 de los 41 niños (92,7\%) el tratamiento inicial fue la enucleación (extirpación del ojo) que se efectuó en forma primaria en 45 de los 59 ojos afectados $(76,27 \%)$ y sólo en 3 niños $(7,31 \%)$ se efectuó quimioreducción inicial antes de la enucleación por tratarse de casos con

Tabla 3. Clasificación de Reese-Ellsworth ${ }^{(*)}$ para 59 ojos de 41 niños con retinoblastoma

\begin{tabular}{lcccc}
\hline Etapa & RB unilateral & $\begin{array}{c}\text { RB bilateral: } \\
\text { Ojo enucleado }\end{array}$ & $\begin{array}{c}\text { RB bilateral: } \\
\text { Ojo conservado }\end{array}$ & Total \\
\hline Ia & 0 & 0 & 4 & 4 \\
Ib & 0 & 0 & 3 & 3 \\
Ila & 0 & 0 & 3 & 3 \\
IIb & 0 & 0 & 0 & 3 \\
IIIa & 0 & 2 & 1 & 5 \\
IIIb & 3 & 2 & 0 & 1 \\
IVa & 0 & 1 & 0 & 1 \\
IVb & 0 & 0 & 0 & 26 \\
Va & 13 & 13 & 0 & 9 \\
Vb & 4 & 5 & 0 & 4 \\
Sin datos & 2 & 2 & 0 & 48 \\
Total enucleaciones & 23 & 25 & 11 & 59 \\
Total ojos & 23 & 25 & & \\
\hline
\end{tabular}

${ }^{*}$ ) Adaptada de Abramson $\mathrm{DH}^{6}$ 
extensión orbitaria que imposibilitaban la enucleación inicial, siendo 2 de estos casos RB bilaterales que fallecieron al poco tiempo después por diseminación de la enfermedad a distancia. Del total de 59 ojos afectados por RB la enucleación fue necesaria en un total de 48 $(81,3 \%)$ durante el período de seguimiento de este estudio, correspondiendo a los 23 niños con RB unilaterales (23 ojos) junto con 18 ojos de los 18 niños con RB bilateral. Los otros 18 ojos de este grupo de tumores bilaterales recibieron diferentes tratamientos adicionales, siendo necesario finalmente enuclear el segundo ojo en 7 de ellos, completándose el total de 48 ojos enucleados. En 17 de 23 niños (73,9\%) con RB unilateral la enucleación fue el único tratamiento necesario para controlar la enfermedad en tanto que en los 6 restantes se requirió de tratamiento adicional complementario, necesitando 4 de ellos quimioterapia para enfermedad extraocular y los otros 2 quimioterapia más radioterapia externa.

\section{Histopatología de Ojos Enucleados}

De los 48 ojos enucleados se logró obtener información histopatológica en 45 (89,58\%). Dieciseis tumores presentaron signos de diferenciación (presencia de rosetas de FlexnerWintersteiner y menos frecuentes rosetas de Homer-Wright), 19 fueron indiferenciados y en 10 no se consignó el grado de diferencia- ción. Se observó que los tumores más diferenciados presentaron menor compromiso de estructuras anatómicas por extensión y tendieron a permanecer intraoculares (12 de 16). Los tumores indiferenciados presentaron un comportamiento más invasor, comprometiendo más estructuras anatómicas oculares y presentando una mayor tendencia (15 de 19) a la extensión extraocular. Tabla 4.

Todos los niños (18) con RB bilateral recibieron enucleación de un ojo y terapias complementarias sistémicas (quimioterapia) y/o múltiples terapias focales para el ojo conservado en forma de LASER (9 ojos, 28,1\%), criotrerapia (7 ojos, 21,9\%), radioterapia externa (10 ojos, $31,3 \%$ ), braquiterapia ( 3 ojos, $9,4 \%$ ) e inyección de quimioterapia subtenoniana (3 ojos, $9,4 \%)$. Siete niños tuvieron que ser enucleados en forma bilateral por falta de respuesta a la terapia mencionada.

\section{Quimioterapia}

Veinticuatro de los 41 niños (58,5\%) recibieron quimioterapia sistémica, administrándose a la totalidad de los 18 pacientes con RB bilateral y a 6 de los 23 casos $(26,1 \%)$ unilaterales enucleados cuando la histopatología demostró enfermedad extraocular. Tabla 5. El resumen de todos los tipos de tratamiento locales y sistémicos recibidos en la totalidad de nuestros pacientes se detalla en la Tabla 6 .

Tabla 4. Estructuras comprometidas y grado de diferenciación histológica en $\mathbf{4 5}$ ojos enucleados

\begin{tabular}{lrrrr}
\hline Extensión del tumor & BD & ID & NC & Total \\
\hline Confinado a retina & 12 & 4 & 7 & 23 \\
Coroides & 0 & 3 & 3 & 6 \\
NO con borde quirúrgico libre & 3 & 2 & 0 & 5 \\
NO + esclera + coroides + CA & 0 & 4 & 0 & 4 \\
Coroides+ NO & 1 & 1 & 0 & 2 \\
Coroides + CA & 0 & 1 & 0 & 1 \\
Esclera & 0 & 1 & 0 & 1 \\
NO con borde quirúrgico comprometido & 0 & 1 & 0 & 1 \\
Coroides + NO + esclera & 0 & 1 & 0 & 1 \\
Cuerpo ciliar & 0 & 1 & 0 & 1 \\
Total ojos & 16 & 19 & 10 & 45 \\
\hline
\end{tabular}

$\mathrm{RB}=$ Retinoblastoma; $\mathrm{CA}=$ Cámara anterior; $\mathrm{NO}=$ Nervio Óptico; $\mathrm{BD}=$ Bien diferenciado; $\mathrm{ID}=$ Indiferenciado; $\mathrm{NC}=$ Diferenciación no consignada en ficha clínica 
Tabla 5. Tipo y número de ciclos de Quimioterapia utilizada en 24 niños con Retinoblastoma

\begin{tabular}{lccccc}
\hline & \multicolumn{5}{c}{ Número de Niños tratados en cada esquema } \\
Tipo quimioterapia & $\mathbf{2}$-5 ciclos & $\mathbf{6}$-7 ciclos & $\mathbf{8}$ ciclos & $\mathbf{>} \mathbf{8}$ ciclos & Total \\
\hline CVE alternada con VID & 0 & 1 & 9 & 1 & 11 \\
CVE & 1 & 7 & 0 & 1 & 9 \\
CVE + adriamicina & 0 & 0 & 0 & 1 & 1 \\
CEV + VID + MTX intratecal & 1 & 0 & 0 & 0 & 1 \\
CVE + VID + MTX y ARA-C intratecal & 0 & 0 & 1 & 0 & 1 \\
CEV + VID + MTX + citarabina intratecal & 0 & 1 & 0 & 0 & 1 \\
Total pacientes & 2 & 9 & 10 & 3 & 24 \\
\hline
\end{tabular}

$\mathrm{C}=$ Carboplatino; $\mathrm{V}=$ Vrincristina; $\mathrm{E}=$ Etopósido; I= Ifosfamida; $\mathrm{D}=$ Doxorrubicina; $\mathrm{MTX}=$ Metotrexato; ARA-C=Citarabina

Tabla 6. Tipos de tratamientos utilizados en 41 niños con Retinoblastoma

\begin{tabular}{|c|c|c|c|c|c|c|}
\hline \multirow{2}{*}{$\begin{array}{l}\text { Tipo de Tratamiento } \\
\text { Sólo Enucleación }\end{array}$} & \multicolumn{2}{|c|}{$\begin{array}{l}\text { RB unilateral } \\
\text { n niños (\%) }\end{array}$} & \multicolumn{2}{|c|}{$\begin{array}{l}\text { RB bilateral } \\
\text { n niños (\%) }\end{array}$} & \multicolumn{2}{|c|}{$\begin{array}{c}\text { Total } \\
\text { n niños (\%) }\end{array}$} \\
\hline & 17 & $(73,9)$ & 0 & $(0)$ & 17 & $(41,5)$ \\
\hline Enucleación + QT + Tx. Focal & 0 & $(0)$ & 7 & $(38,9)$ & 7 & $(17,1)$ \\
\hline Enucleación + QT & 4 & $(17,4)$ & 2 & $(11,1)$ & 6 & $(14,6)$ \\
\hline Enucleación + QT + RT & 2 & $(8,7)$ & 4 & $(22,2)$ & 6 & $(14,6)$ \\
\hline Enucleación + QT + RT + Tx. Focal & 0 & (0) & 5 & $(27,8)$ & 5 & $(12,2)$ \\
\hline Sólo Quimioterapia & 0 & $(0)$ & 0 & $(0,0)$ & 0 & $(0,0)$ \\
\hline Total & 23 & $(100)$ & 18 & $(100)$ & 41 & $(100)$ \\
\hline
\end{tabular}

QT= Quimioterapia sistémica; RT = Radioterapia; Tx. Focal = LASER y/o Criopterapia y/o carboplatino subconjuntival

\section{Recurrencias tumorales y aparición de nuevos tumores}

Sólo un caso de RB unilateral se convirtió posteriormente en bilateral por compromiso del ojo sano a los 8 meses desde el primer diagnóstico. En 4 niños los tumores recidivaron en su localización inicial a pesar del tratamiento focal y con quimioterapia, ocurriendo la recurrencia durante el tratamiento con quimioterapia en un caso y una vez finalizada ésta en 3 . En otros 4 niños aparecieron nuevos tumores en diferentes localizaciones dentro del mismo ojo, ocurriendo en 3 casos durante la quimioterapia y en un caso una vez finalizada ésta.

\section{Mortalidad, extensión extraocular y metástasis a distancia}

Durante el período de seguimiento del presente estudio fallecieron 5 niños de sexo masculino $(12,2 \%)$, siendo el promedio de su sobrevida de 21,5 meses (rango 6-36). Uno de estos niños presentaba RB trilateral (RB bilateral asociado a pinealoma), 2 tuvieron extensión orbitaria, uno de ellos con compromiso de médula ósea, y 2 presentaron compromiso de nervio óptico y coroides, ambos con metástasis cerebrales y uno de ellos con compromiso del cuerpo ciliar.

\section{Discusión}

El conocer nuestra realidad nacional actualizada en retinoblastoma respecto a sus características clínicas, las modalidades terapéuticas utilizadas y la respuesta a dicho tratamiento es de vital importancia tanto para la oncología pediátrica como para los programas de salud pública a nivel nacional. Si bien hasta la fecha no se dispone de un registro nacional de cáncer que permita conocer en detalle la procedencia de cada uno de los casos de RB de nuestro país, nuestra muestra es altamente representa- 
tiva tanto de la situación epidemiológica como del abordaje terapéutico multidisciplinario del $\mathrm{RB}$ ya que se trata de una heterogénea serie de casos de pacientes referidos desde distintos puntos del país a un hospital del nivel terciario de atención del PINDA durante un número significativo de años.

Globalmente nuestros resultados confirman una preocupante realidad: nuestros pacientes solicitan evaluación médica sólo cuando la enfermedad está ya muy avanzada, hecho que queda reflejado tanto en la edad tardía de consulta ( 21,2 meses en promedio), en el alto número de niños que requirieron enucleación como modalidad de tratamiento inicial $(92,7 \%)$ y en el alto porcentaje de pacientes con tumores unilaterales que además de ser enucleados requirieron quimioterapia complementaria por presentar extensión extraocular de la enfermedad $(26,1 \%)$. A pesar de estos aspectos negativos la sobrevida a 3 años de nuestros pacientes durante el período de seguimiento fue de un $84,8 \%$ siendo comparable a la reportada internacionalmente $^{8-10}$. Los 5 niños que fallecieron lo hicieron dentro de los primeros 3 años de seguimiento, presentando todos ellos diseminación extraocular de la enfermedad. Una consulta precoz cuando el tumor es de pequeño tamaño y por lo tanto, susceptible de ser tratado con terapia focal en modalidad de LASER y/o crioterapia y/o quimioreducción sería el escenario ideal para intentar salvar a estos pacientes y sus ojos. Los esfuerzos para lograr (aunque sea en parte) sospechar y detectar la presencia de retinoblastoma en etapas precoces debe comenzar por el conocimiento y la difusión sobre la existencia de esta enfermedad y la utilidad del examen del rojo pupilar como herramienta diagnóstica para el personal de la salud aplicable desde el nacimiento en el examen de los niños, en el control del niño sano y en toda consulta pediátrica en general. Del mismo modo el alertar a la opinión pública mediante campañas educativas sobre los signos más frecuentes de presentación del retinoblastoma encontrados en nuestro estudio, leucocoria y estrabismo, deberían cada vez más ayudar a alertar precozmente a los padres y tutores de estos niños a consultar a la brevedad.

Nuestros resultados demuestran también que nuestros pacientes han recibido un tratamiento de altísima calidad tanto desde el punto de vista oncológico como oftalmológico. Los niños atendidos en este período recibieron evaluación, tratamiento y seguimiento a cargo de pediatras oncólogos expertos en retinoblastoma y quimioterapia de primera línea de acuerdo a los protocolos del PINDA, en tanto que desde el punto de vista oftalmológico el tratamiento recibido por nuestros niños es en la actualidad el mismo utilizado en centros de referencia internacional, incluyendo la enucleacón y rehabilitación protésica, la fotocoagulación LASER, la crioterapia, inyección periocular de quimioterapia y la radioterapia en placa (braquiterapia) que se practicaron en forma única o combinada a los 18 ojos conservados de nuestros pacientes. De gran utilidad resultó la adquisición por parte de nuestro hospital durante los últimos 2 años de los equipos e instrumental necesarios para cumplir con dicho objetivo sin tener que derivar a nuestros pacientes al sistema privado de salud para el tratamiento focal de los tumores como solía ocurrir frecuentemente previo a la implementación completa de nuestro pabellón de oftalmología. Nuestros resultados también ponen en evidencia la imperiosa necesidad de contar con un sistema de recolección de imágenes tipo Retcam ${ }^{11} \mathrm{u}$ otros que facilitan grandemente el tratamiento, seguimiento y documentación fotográfica de los tumores, permitiendo además compartir los casos complejos con otros expertos en el tema mediante telemedicina.

Nuestra revisión pone de manifiesto varias e importantes falencias en la documentación clínica de nuestros pacientes, propio de los estudios retrospectivos no controlados, pero que nos alertan sobre cómo advertir y mejorar a futuro este aspecto del manejo. Nuestras fichas carecían de un registro cuidadoso sobre el examen de los familiares de niños con RB en al menos la mitad de los casos lo que puede haber influído en el bajo número de familiares afectados con mayor probablilidad de enfermedad hereditaria encontrados en nuestra revisión. El diseñar y manejar fichas clínicas que incluyan la descripción cuidadosa de los árboles genealógicos, el examen de fondo de ojos de todos los familiares de riesgo e idealmente el estudio genético molecular de cada uno de los casos 
podrían ayudar a conocer mejor la realidad del $\mathrm{RB}$ hereditario en nuestro país.

Aunque nuestros pacientes recibieron un estricto protocolo de seguimiento oncológico y oftalmológico con controles y exámenes complementarios tanto en policlínico como en pabellón bajo anestesia general, resultó muy difícil obtener información sobre los resultados visuales de los pacientes que conservaron alguno de sus ojos. La toma de visión en población pediátrica pre-verbal (la mayoría de los pacientes con retinoblastoma) es en general de muy difícil cuantificación por lo que exámenes como el estudio de la mirada preferencial (test de Teller ${ }^{\circledR}$ ) podrían resultar de gran utilidad para intentar conocer la visión de nuestros pacientes antes, durante y después del tratamiento ${ }^{12}$. La pronta implementación de esta técnica en nuestro hospital será de gran ayuda para el seguimiento de estos niños. Por último, la necesidad de implementar la clasificación internacional para el retinoblastoma tanto en nuestro hospital como en el resto de los centros oncológicos del mundo se está haciendo cada vez más imperiosa en una era en que el tratamiento con quimioterapia y terapia focal son el standard de calidad para esta enfermedad ${ }^{7}$.

\section{Conclusiones}

El retinoblastoma constituye una grave enfermedad con múltiples secuelas que incluyen desde el compromiso variable de visión hasta la pérdida del globo ocular y la vida. En los pacientes atendidos en nuestro medio hemos observado grandes contrastes: Mientras su sobrevida alcanza niveles comparables a la de paises desarrollados existen aún grandes desafíos por lograr para alcanzar un nivel óptimo en el tratamiento de esta patología tales como disminuir el elevado número de niños que requieren enucleación, que consultan con un grado de enfermedad muy avanzada, que requieren de quimioterapia y/o radioterapia externa como terapias complementarias a la enucleación y que se presentan con extensión extraocular de la enfermedad. Todas estas preocupantes situaciones observadas en los niños con RB nuestro medio tienen en común estar asociadas a una consulta tardía. La educación tanto del personal de la salud como de la población en general respecto a la utilidad del examen del rojo pupilar y la consulta precoz frente a sospecha de leucocoria o estrabismo podrían contribuir a la obtención de aún mejores resultados en el tratamiento de nuestros pacientes con RB.

Esperamos que la información contenida en nuestro reporte respecto a la realidad nacional sobre el retinoblastoma resulte de utilidad para la comunidad médica, científica y, más importantemente, para los niños de Chile.

\section{Referencias}

1.- Seregard S, Lundell G, Svedberg H, Kivela T: Incidence of retinoblastoma from 1958 to 1998 in Northern Europe: advantages of birth cohort analysis. Ophthalmology 2004; 111: 1228-32.

2.- Ferris FL, 3rd, Chew EY: A new era for the treatment of retinoblastoma. Arch Ophthalmol 1996;114:1412.

3.- Marchevsky S, Beresi V, Quintana J, del Pozo H, Charlin C: Retinoblastoma. Rev Chil Pediatr 1979; 50: 32-4.

4.- Sepulveda LE, Beresi V, Quintana J, del Pozo H: Second cancer in pediatric patients. Rev Chil Pediatr 1990; 61: 82-6.

5.- Musarella MA, Gallie BL: A simplified scheme for genetic counseling in retinoblastoma. J Pediatr Ophthalmol Strabismus 1987; 24: 124-5.

6.- Abramson DH, Schefler AC: Update on retinoblastoma. Retina 2004; 24: 828-48.

7.- Shields CL, Shields JA: Basic understanding of current classification and management of retinoblastoma. Curr Opin Ophthalmol 2006; 17: 228-34.

8.- MacCarthy A, Draper GJ, Steliarova-Foucher E, Kingston JE: Retinoblastoma incidence and survival in European children (1978-1997). Report from the Automated Childhood Cancer Information System project. Eur J Cancer 2006; 42: 2092-102.

9.- Ozkan A, Pazarli H, Celkan T, et al: Retinoblastoma in Turkey: survival and clinical characteristics 19812004. Pediatr Int 2006; 48: 369-73.

10.- Sanders BM, Draper GJ, Kingston JE: Retinoblastoma in Great Britain 1969-80: incidence, treatment, and survival. Br J Ophthalmol 1988; 72: 576-83.

11.- Lee TC, Lee SW, Dinkin MJ, Ober MD, Beaverson KL, Abramson DH: Chorioretinal scar growth after 810 nanometer laser treatment for retinoblastoma. Ophthalmology 2004; 111: 992-6.

12.- Ross G, Lipper EG, Abramson D, Preiser L: The development of young children with retinoblastoma. Arch Pediatr Adolesc Med 2001; 155: 80-3. 